\title{
Adaptive Dynamic Modeling and Simulation for Variable Cycle Engine
}

\author{
Feng Lu*, Zelong Zou and Jinquan Huang \\ Jiangsu Key Laboratory of Aerospace Power System, Nanjing University of Aeronautics and \\ Astronautics, 210016, China \\ ${ }^{*}$ Corresponding author
}

\begin{abstract}
Keywords: Variable cycle engine, Component level model, Health estimation, Adaptive model.
\end{abstract}
\begin{abstract}
Advanced aircraft engine needs higher unit thrust and lower fuel consumption nowadays. The variable cycle engine (VCE) could meet these requirements, and it becomes the top choice of the new generation aircraft. It is a difficult task to find a satisfactory dynamic model that can well match the VCE during its life time due to the performance deterioration with the increase of service time. Besides, the more variables of control and actuator are involved. This paper presents an adaptive dynamic modeling method for the VCE based on the state estimation. It mainly contains the nonlinear component level modeling of VCE and self-tuning dynamic model combined with a distributed extended Kalman filter. The digital simulations are carried out to verify the effectiveness of the VCE modelling methodology.
\end{abstract}

\section{Introduction}

The turbofan engine is more fuel-efficient than turbojet engine as Mach number is less than one. When the flight speed is greater than $1.8 \mathrm{Ma}$, the thrust advantage of turbojet engine is more obvious. These two independent types of engines hardly meet the requirement of the latest generation of aircraft power system, and variable cycle engines (VCE) become one of the appropriate candidates [1]-[2].

Different from turbojet engine or turbofan engine, the VCE runs in different working modes in a fairly wide flight envelope according to different tasks. It achieves the goal of less fuel consumption during cruising and higher thrust at the acceleration process. Thus it easily satisfies the needs of advanced aircraft to perform a variety of tasks under complex flight conditions. In order to shorten the research cycle and reduce the manufacture costs of the VCE, it is necessary to master the dynamic model with high enough accuracy in the course of lifetime. In recent years, the componentlevel modeling technology is well developed for aircraft engine and gas turbine engine ${ }^{[3]-[4]}$.

However, the compressor characteristics of the VCE vary significantly due to the deterioration, and it makes the initial dynamic model using the component-level modeling technology based on aero-thermodynamic principle deviate from the actual engine output. The compressor feature parameters, such as flow capacity and efficiency, are usually employed to represent the compress maps. The feature parameters are unmeasurable, the variations of which can be reflected by the sensor measurements. Thus we can try to tune the engine model outputs from the change of the unmeasurable feature parameters. For these purposes, the self-tuning dynamic model of the VCE is developed. It includes the nonlinear component level model and Kalman filter to estimate the state parameters of component characteristics. In the simulation, the modelling methodology is evaluated in the steady and transient behaviour at six working conditions of the VCE.

The remaining parts of the paper is organized as follows. The nonlinear component level model of the VCE is built up in Section II. Section III presents the adaptive dynamic model of the VCE, and it includes the state estimator based on a distributed Kalman filter. The simulation and analysis are given in Section IV. The conclusion is draw in Section V. 


\section{Component Level Modeling of the VCE}

\section{Component Model of VCE}

An advanced VCE owns three additional key components of core drive fan stage (CDFS), core drive fan stage bypass and sub-duct, compared to the conventional turbofan engine ${ }^{[5]}$. The input of VCE model is Height $H$, Mach number $M a$, sea level atmospheric pressure $P_{0}$, temperature $T_{0}$, combustor fuel supply $W_{f b}$, afterburner fuel supply $W_{f a}$, mode valve area $A_{114}$, front adjustable duct ejector area $A_{224}$, rear adjustable duct ejector area $A_{163}$, rear mixing chamber duct inlet area $A_{6}$, nozzle throat area $A$, nozzle outlet area $A$, the angle of fan inlet guide vane is $\alpha_{F}$, the angle of CDFS inlet guide vane is $\alpha_{C D F S}$, the angle of compressor inlet guide vane is $\alpha_{C}$, the installation angle of high pressure turbine guide vane is $\alpha_{m}$ and the installation angle of low pressure turbine guide vane is $\alpha_{L T}$. Variable cycle engine structure is shown in figure I.

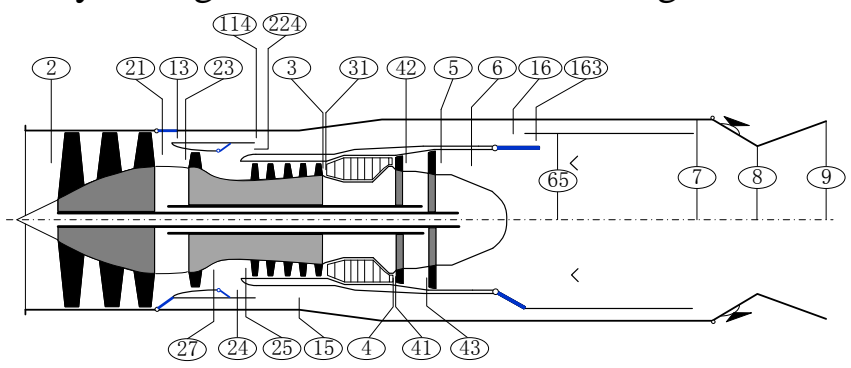

Figure 1. GAS path component structure of the VCE

The high pressure compressor can further increase the air temperature and pressure, so that it is more easily to be ignited in combustor. Since the different rotating components follow the similar operating mechanisms, the mathematical model of the compressor is only presented in detail in the paper.

(1) Calculate the compressor corrected speed $n_{H, \text { cor }}=\frac{n / \sqrt{T_{\text {in }}}}{n_{d} / \sqrt{T_{\text {ind }}}}$.

(2) The import corrected flow $W_{\text {cor }}$ and import corrected efficiency $\eta_{\text {cor }}$ is obtained from the compressor maps. The actual physical values are then deduced by the inverse calculation of correction:

$$
\left\{\begin{array}{l}
W_{\text {cor }}=f_{5}\left(\alpha_{C}, n_{\text {cor }}, \pi\right) \\
\eta_{c o r}=f_{6}\left(\alpha_{C}, n_{\text {cor }}, \pi\right) \\
W_{\text {in }}=C_{m} W_{c o r} \frac{P_{\text {in }}}{P_{\text {ind }}} \sqrt{\frac{T_{\text {ind }}}{T_{\text {in }}}} \llbracket S W_{3} \\
\eta=C_{\eta} \eta_{c o r} \llbracket S E_{3}
\end{array}\right.
$$

where $f_{5}()$ and $f_{6}()$ are the interpolation functions of flow capacity and efficiency from pressure ratio and corrected speed. $S E_{3}$ and $S W_{3}$ are the compressor feature coefficients of efficiency and flow capacity. $S E_{3}$ and $S W_{3}$ are equal to one as no deterioration.

(3) Calculate outlet entropy Smid, enthalpy $\mathrm{H}_{\text {out }}$ and surge margin SM are as follows:

$$
\left\{\begin{array}{l}
S_{\text {mid }}=S_{\text {in }}+\lg \pi \\
H_{\text {out }}=H_{\text {in }}+\left(H_{\text {ind }}-H_{\text {in }}\right) / \eta \\
S M=\left(\pi_{\text {surg }} / W_{\text {surg }}\right) /\left(\pi / W_{\text {cor }}\right)-1
\end{array}\right.
$$

(4) The release ratio of compressor inlet air is $r_{D} \%$. The stage of compressors is $r$, and air release position is at $k$ stage labelled the $j$-th $(j<r)$ level. The release volume $W_{D k}$, total pressure $P_{D k}$, entropy $S_{\text {indDk, }}$ and enthalpy $H_{\text {outDK }}$ are obtained: 


$$
\left\{\begin{array}{l}
W_{D k}=W_{i n} r_{D k} \% \\
P_{D k}=P_{i n} \pi_{D k} \\
S_{\text {indDk }}=S_{\text {in }}+\lg \pi_{D k} \\
H_{\text {outDk }}=H_{\text {in }}+\left(H_{\text {indDk }}-H_{\text {in }}\right) / \eta_{D k}
\end{array}\right.
$$

where the release pressure ratio is $\pi_{D k}=\pi^{j / r}$ and efficiency $\eta_{D k}=\eta$. Thus exhaust air is related to total temperature $T_{D k}$ and power $N_{D k}$ are as follows:

$$
\left\{\begin{array}{l}
T_{D k}=f_{H 2 T}\left(F F, H_{\text {outDk }}\right) \\
N_{D k}=W_{D k}\left(H_{\text {outDk }}-H_{\text {in }}\right)
\end{array}\right.
$$

(5) The calculation procedure of the rest air bleed and induction is the same as Eq.(3) and Eq.(4), so it is no longer discussed. The outlet pressure, temperature, flow capacity and power are calculated by:

$$
\left\{\begin{array}{l}
P_{\alpha u}=P_{\text {in }} \pi \\
T_{\text {out }}=H_{H 2 T}\left(F F, H_{\text {out }}\right) \\
W_{\text {out }}=W_{\text {in }}-\sum_{l=1}^{q} W_{a}-\sum_{k=1}^{m} W_{D k} \\
N_{c}=W_{\text {out }}\left(H_{\alpha t}-H_{i n}\right)+\sum_{l=1}^{q} N_{d}+\sum_{k=1}^{m} N_{D k}
\end{array}\right.
$$

where $W_{C l}$ and $N_{C l}$ are the flow capacity and power of air induction for cooling hot-section components.

\section{Common Operating Equations of the VCE}

(1) The mass flow balance equations are as follows:

The mass flow of fan outlet balance:

$$
W_{2}-W_{21}-W_{13}=0
$$

where $W_{2}$ is the mass flow of fan inlet, $W_{21}$ is the mass flow of CDFS inlet, and $W_{13}$ is to the bypass depended on the mode selection valve. The mass flow of combustor outlet balance:

$$
W_{g 41}-W_{a 3}-W_{f}-W_{h t, c o o l N G V}=0
$$

where $W_{g 41}$ is the mass flow of HPT inlet, $W_{a 3}$ is the compressor outlet, $W_{f}$ is the fuel supply in combustion, $W_{h t, c o o l N G V}$ is air mass flow to cool HPT guide vane. The mass flow of HPT outlet balance:

$$
W_{g 43}-W_{a 3}-W_{f}-W_{h t, c o l l N G}-W_{h t, \text { colladade }}=0
$$

where $W_{g 43}$ is the mass flow of LPT inlet, and $W_{h t, \text { coolilade }}$ is the air mass flow to cool HPT blade. The mass flow of mixer outlet balance:

$$
W_{g 7}-W_{g 9}=0
$$

(2) The static pressure balance are as follows:

The static pressures between two ducts at the front duct ejector outlet, and two ducts at the rear duct ejector outlet:

$$
\begin{aligned}
& P_{s 114}-P_{s 224}=0 \\
& P_{s 16}-P_{s 65}=0
\end{aligned}
$$

where $P_{\text {s114 }}$ is the static pressure of CDFS outlet, and $P_{s 224}$ is the static pressure of mode selcetor valve outdoor. $P_{s 16}$ and $P_{s 65}$ are the static pressure of the bypass outlet and LPT outlet.

(3) The spool power balance are as follows:

The dynamics equation of low and high pressure spool: 


$$
\begin{aligned}
& N_{L T} \eta_{m L}-N_{F}-\left(\frac{\pi}{30}\right)^{2} J_{L} n_{L} \frac{d n_{L}}{d t}=0 \\
& N_{H T} \eta_{m H}-N_{C D}-N_{C}-N_{E X T}-\left(\frac{\pi}{30}\right)^{2} J_{H} n_{H} \frac{d n_{H}}{d t}=0
\end{aligned}
$$

where $N_{L T}$ and $N_{H T}$ are the generated powers from LPT and HPT. $\eta_{m L}$ and $\eta_{m L}$ is the mechanical efficiencies of low-pressure and high-pressure spools. $N_{F}, N_{C D}, N_{C}$, and $N_{E X T}$ are the power consumptions of fan, CDFS compressor and accessories. $J_{L}$ and $J_{H}$ are rotor inertias of low pressure and high pressure spools. The involved nonlinear equations from Eq. (6) to Eq. (11) are solved by Newton-Raphson method.

\section{Adaptive VCE Model}

\section{VCE Model Combined with State Estimator}

In the course of VCE service, foreign damage, corrosion of wet air, wear of blade tips and casing, burning loss of turbine blades, warping deformation of hot-end parts, fouling of components and other factors will cause the performance deterioration of the VCE. The efficiency and flow capacity of rotor components, such as fan, CDFS, compressor, HPT and LPT used to depict performance deterioration, which in turn lead to the avaliable measurements like speed, temperature and pressure. Therefore, the magnitudes of engine gas path deterioration are obtained from the sensor measurements, and the state estimator could provide a bridge between the unmeasurable feature parameters and sensor parameters.

The VCE rotor components includes fan, CDFS, compressor, HPT and LPT, the feature parameters $S E_{i}$ and $S W_{i}$ of these examined parameters are defined:

$$
S E_{i}=\frac{\eta_{i}}{\eta_{i}^{*}}, S W_{i}=\frac{W_{i}}{W_{i}^{*}}
$$

where $\eta_{i}$ and $\eta_{i}^{*}$ are real and ideal component efficiencies, and $W_{i}$ and $W_{i}^{*}$ are real and ideal component mass flows. Thus the feature vectors of the VCE is given $\boldsymbol{h}=\left[S E_{1} S W_{1} S E_{2} S W_{2} S E_{3} S W_{3}\right.$ $\left.S E_{4} S W_{4}\right]^{\mathrm{T}}$, wherein subscripts $i$ from 1 to 4 separately relate to fan, CDFS, compress and HPT.

The feature parameters are tuned to reach the consistency between the actual sensor measuremnts and the model outputs. Since the VCE model is nonlinear, distributed extended Kalman filter (DEKF) algorithm is utilized to state estimation. As a result, an adaptive VCE model is developed from the conventional componnet level model, and combined with the DEKF. The residuals between the estimated from the VCE and its model drive the DEKF to compute the rotating compoents' feature parameters. The framework of the adaptive VCE model is shown in Figure 2.

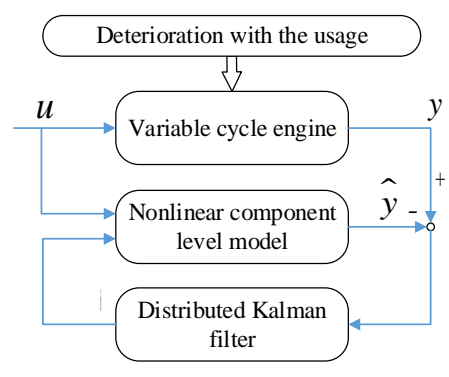

Figure 2. Adaptive VCE model combined with dekf

\section{Distributed Extended Kalman Filter}

The noninear component level model of the VCE is packaged to a dynamic link library, and the discrete mathematical expression is given:

$\boldsymbol{x}_{k+1}=f\left(\boldsymbol{x}_{k}, \boldsymbol{u}_{k}\right)+\boldsymbol{w}_{k}$
$\boldsymbol{y}_{k}=g\left(\boldsymbol{x}_{k}, \boldsymbol{u}_{k}\right)+\boldsymbol{v}_{k}$ 
where $\boldsymbol{u}_{k}$ is the input vector at step $k$, and $\boldsymbol{u}=\left[W_{f} A_{8} A_{224} A_{163}\right]$. The four elements are fuel flow $W_{f}$, nozzle throat area $A_{8}$, front adjustable duct ejector area $A_{224}$ and rear adjustable duct ejector area $A_{163}$. The state vector $\boldsymbol{x}=\left[n_{L} n_{H} S E_{1} S W_{1} S E_{2} S W_{2} S E_{3} S W_{3} S E_{4} S W_{4}\right]$, and it contains component feature parameters except for two spool speeds. The sensor measurements are $y=\left[n_{L} n_{H} T_{21} P_{21} T_{15}\right.$ $P_{15} T_{3} P_{3} T_{5} P_{5}$, wherein $T_{21}$ is the fan outlet temperature, $P_{21}$ is the fan outlet pressure, $T_{15}$ is the CDFS outlet temperature, $P_{15}$ is the CDFS outlet pressure, $T_{3}$ is the compressor outlet temperature, $P_{3}$ is the compressor outlet pressure, $T_{5}$ is the LPT outlet temperature, $P_{5}$ is the LPT outlet pressure.

$\boldsymbol{W}_{k}$ and $\boldsymbol{V}_{k}$ are the process noise and measurement noise, and $\boldsymbol{Q}$ and $\boldsymbol{R}$ are related to their covariance matrix, respectively. These noise follows Gauss white noise. All measurements are divided into four groups based on the sensors' locations, and that is to say, [ $n_{L} n_{H} T_{21} P_{21}$ ], [ $n_{L} n_{H}$ $\left.T_{15} P_{15}\right],\left[n_{L} n_{H} T_{3} P_{3}\right]$ and $\left[n_{L} n_{H} T_{5} P_{5}\right]$. The procedure of DEKF algorithm is given as follows.

(1) The state variables and covariance matrix are initialized:

$$
\left\{\begin{array}{l}
Q_{i}=\beta_{i}^{-1} Q \\
P_{i, 0}=\beta_{i}^{-1} P_{0} \quad i=1,2, \cdots, N \\
\hat{x}_{i, 0}=\hat{x}_{0}
\end{array}\right.
$$

where $x_{0}$ is the initial global state variables, $P_{0} \mathrm{~s}$ the initial error covariance matrix. $N$ is the number of groups in the DEKF, and $\beta_{i}=1 / N$.

(2) The update computation of local filter at step $k$ :

$$
\left\{\begin{array}{l}
\hat{x}_{i, k k-1}=f\left(X_{i, k-1}, u_{i, k-1}\right) \\
P_{i, k k-1}=A P_{i, k-1} A^{T}+Q \\
K_{i, k}=P_{i, k k-1} C^{T}\left(C_{i} P_{i, k k-1} C_{i}^{T}+R_{i}\right)^{-1} \\
\hat{X}_{i, k}=\hat{X}_{i, k k-1}+K_{i, k}\left[y_{i, k}-g_{i}\left(\hat{X}_{i, k k-1}, u_{i, k}\right)\right] \\
P_{i, k}=\left(I-K_{i, k} C_{1}\right) P_{i, k k-1}
\end{array}\right.
$$

where Jacobian matrices $\boldsymbol{A}$ and $\boldsymbol{C}_{i}$ are calculated:

$A=\frac{\partial f\left(x_{k-1}, u_{k-1}\right)}{\partial x_{k-1}}, C_{i}=\frac{\partial g_{i}\left(x_{i, k}, u_{i, k}\right)}{\partial x_{i, k}}$

(3) Information fusion of local filters and the share of information in the master filter are given:

$$
\begin{aligned}
& \left\{\begin{array}{l}
P_{k}^{-1}=\sum_{i=1}^{N} P_{i, k}^{-1} \\
\hat{x}_{k}=P_{k} \sum_{i=1}^{N}\left(P_{i, k}^{-1} \hat{x}_{i, k}\right)
\end{array}\right. \\
& \left\{\begin{array}{l}
P_{i, k}=\beta_{i}^{-1} P_{k} \\
\hat{x}_{i, k}=\hat{x}_{k}
\end{array}\right.
\end{aligned}
$$

During the state estimiation process, step (2) to step (4) are iteratively executed.

\section{Simulation and Analysis}

The involved VCE model is evaluated in the six operating conditions in the simulation ${ }^{[6]}$. Ten gas path failure modes are added to the healthy VCE model to simulate performance deterioration. Table I gives the typical operating conditions of the VCE, and Table II shows the gas path failure modes.

Table 1. VCE operating point

\begin{tabular}{ccccccc}
\hline Labels & a & b & c & d & e & f \\
\hline Flight & $H=0$, & $H=0$, & $H=11 \mathrm{~km}$, & $H=11 \mathrm{~km}$, & $H=10 \mathrm{~km}$, & $H=0$, \\
conditions & $M a=0$ & $M a=0$ & $M a=1.5$ & $M a=1.7$ & $M a=2$ & $M a=0$ \\
Operating & Single & $\begin{array}{c}\text { Double } \\
\text { modes }\end{array}$ & Single & Single & Single & Mode \\
mode & mode & mode & mode & switch \\
\hline
\end{tabular}


Table 2. Gas path failure modes of the VCE.

\begin{tabular}{|c|c|c|c|c|c|c|c|c|c|c|}
\hline Labels & I & II & III & IV & V & VI & VII & VIII & IX & $\mathbf{X}$ \\
\hline h & $S E_{1}-1$ & $-0.5, S W_{1^{-}}$ & $S E_{2}-1$ & $\begin{array}{l}S W_{2}-1, \\
S E_{2}-0.7\end{array}$ & $S W_{2}-1$ & $S E_{3}-1$ & $S W_{3}-1$ & $\begin{array}{c}S E_{3}-0.7 \text {, } \\
S W_{3}-1\end{array}$ & $S E_{4}-1$ & $S W_{4}+1$ \\
\hline
\end{tabular}

\section{Steady Behavior}

The VCE runs at the operating points from a to e in Table I which relate to the steady behavior, and the corrected $n_{H}$ is equal to $100 \%$. Figure III shows the estimated state variables and outputs from the adaptive VCE model at the operating condition 4. The gas path deteration mode VIII is introduced to the VCE at $6 \mathrm{~s}$.

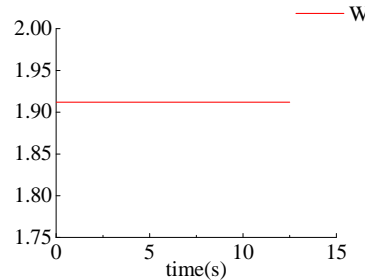

(a) $W_{\mathrm{fb}}$

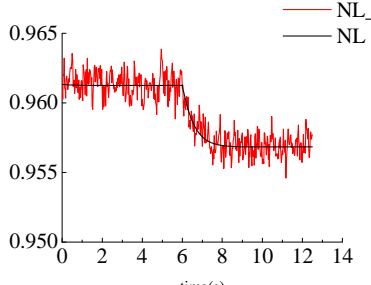

(c) $n_{L}$

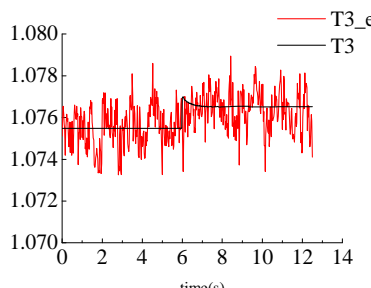

(e) $T_{3}$

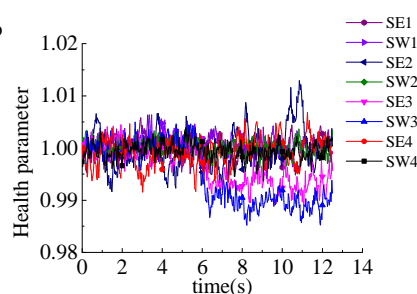

(b) $\boldsymbol{h}$

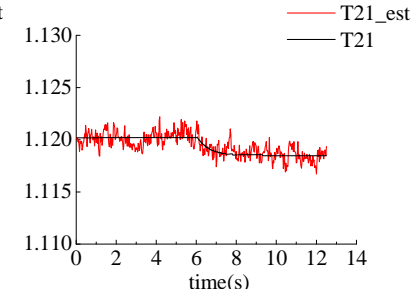

(d) $T_{21}$

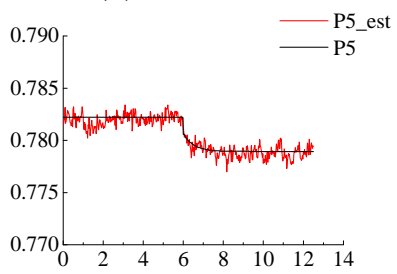

(f) $P_{5}^{\text {tin }}$

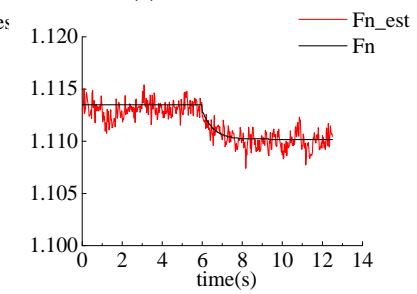

(h) $F_{n}$

Figure 3. The adaptive VCE model tracking results at the operating condition 4

In Figure III(h), the relative error mean of thrust is about $0.061 \%$, the average value of absolute error is 0.00068 , and the maximum deviation is 0.00285 . The estimated $F_{n}$ fluctuation is small around its real value. Besides, the estimated feature parameters of the examined components also move around the actual deteriorated status at the operating condition 4 in the steady behavior. In addition, the performance index of root mean square error, called RMSE, is introduced to reveal the model tracking accuracy. Table III gives the RMSE of adaptive VCE model output at the operating conditions from a to e in steady states. As seen from Table III, the RMSE are all below 0.03, and it indicates the adaptive VCE model could provide satisfactory tracking accuracy during performance deterioration. 
Table 3. RMSE of the adaptive VCE model in steady states

\begin{tabular}{|c|c|c|c|c|c|c|c|c|c|c|}
\hline \multirow{2}{*}{$\begin{array}{l}\text { Operating } \\
\text { conditions }\end{array}$} & \multicolumn{10}{|c|}{ Gas path deterioratin modes } \\
\hline & $I$ & II & III & $I V$ & $V$ & $V I$ & VII & VIII & $I X$ & $X$ \\
\hline a & 0.0161 & 0.0171 & 0.0172 & 0.0162 & 0.0162 & 0.0175 & 0.0177 & 0.0181 & 0.0182 & 0.0168 \\
\hline b & 0.0205 & 0.0224 & 0.0238 & 0.0239 & 0.0199 & 0.0269 & 0.0214 & 0.0283 & 0.026 & 0.0255 \\
\hline c & 0.0182 & 0.0206 & 0.0193 & 0.0194 & 0.0205 & 0.0188 & 0.0194 & 0.0191 & 0.0180 & 0.0182 \\
\hline d & 0.0192 & 0.0192 & 0.0200 & 0.0176 & 0.0184 & 0.0191 & 0.0201 & 0.0177 & 0.0197 & 0.0186 \\
\hline e & 0.0170 & 0.0185 & 0.0218 & 0.0176 & 0.019 & 0.0204 & 0.0174 & 0.0187 & 0.0174 & 0.0181 \\
\hline
\end{tabular}

\section{Transient Behavior}

As was mentioned earlier, the most interesting speciality of VCE is that the engine could operates both in the turbofan and turbojet modes. There are two ducts as the VCE works in the turbofan mode, and the air flows to both of the bypass and core ducts. The turbojet mode is related to the single core duct. In this paper, the mode switch process is examined from the double ducts to single duct, and then moves back to double ducts. The fuel flow varies during the mode switch process, and it is recognized as the transient behavior. The simulation time is $50 \mathrm{~s}$, and the sudden gas path degrdation mode I is added at $5 \mathrm{~s}$. The turbofan mode converts to the turbojet mode at $10 \mathrm{~s}$, and it lasts 3s. As 30s, the VCE changes from the single duct to double ducts mode, and it also lasts 3s. The simulation results are shown in Figure IV.

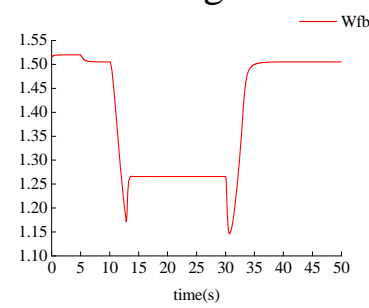

(a) $W_{f b}$

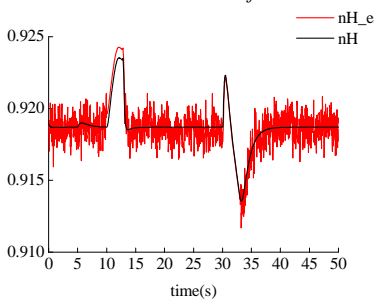

(c) $n_{H}$

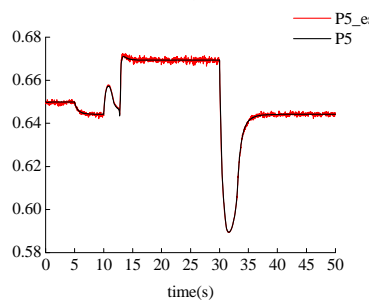

(e) $P_{5}$

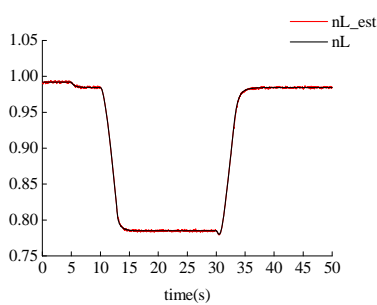

(b) $n_{L}$

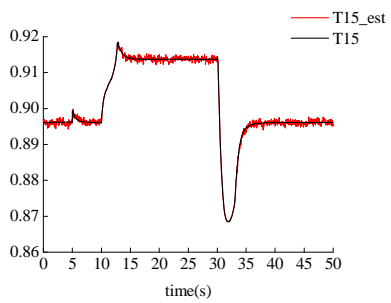

(d) $T_{15}$

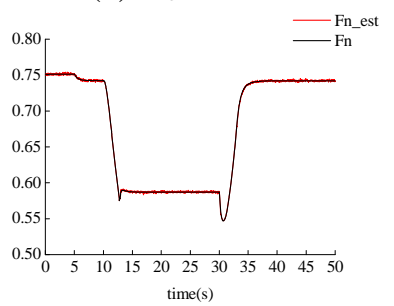

(g) $F_{n}$

Figure 4. The adaptive VCE model tracking results in the transient behavior

From Figure IV(c), the relative error mean of high spool speed is about $0.088 \%$ from 10 s to13s, and the maximum of absolute error is 0.00132. It indicates that the adaptive VCI model track the actual VCE output well during the mode switch process that is from the double ducts to single duct. The relative error mean of $n_{H}$ is about $0.023 \%$, and the maximum of absolute error is 0.00104 from 30s to 33s in Figure IV(c). Throughout the whole simulation process, the relative error mean of $n_{H}$ is about $0.069 \%$. When it comes to the remaining physical parameters, we find that the estimates from the VCE could also closely track their real curves. Hence, it illustrates the adaptive VCE model could generate the satisfactory tracking accuracy during the mode switch process in the transient behavior. 


\section{Conclusion}

DEKF algorithm is designed and applied to build up the adaptive VCE model. The objective is to provide an adaptive VCE model to track the real engine with high enough confidence as performance deterioration during its life time. The modeling methodology consists of nonlinear component level model and state estimator of DEKF. The simulation is carried out on the VCE in steady and transient behaviors, and the results indicate that the adaptive could provide the accurate state estimates and outputs. It confirms the effectiveness of the adaptive dynamic modeling way.

\section{References}

[1] Volponi A.J, “Gas turbine engine health management past, present and future trends," ASME Journal of Engineering for Gas Turbines and Power, 2014, 136(5), p.051201.

[2] Tahan M., Tsoutsanis E., Muhammad M., Karim Z.A.A, "Performance-based health monitoring, diagnostics and prognostics for condition-based maintenance of gas turbines: A review,” Applied Energy, 2017, 198, 122-144.

[3] Pourbabaee B., Meskin N., Khorasani K, "Sensor fault detection, isolation, and identification using multiple-model-based hybrid Kalman filter for gas turbine engines," IEEE Transactions on Control Systems Technology, 2016, 24(4), 1184-1200.

[4] Netto M., Mili L, “A robust data-driven Koopman Kalman filter for power systems dynamic state estimation,” IEEE Transactions on Power Systems, 2018, 33(6), 7228-7237.

[5] Caballero Águila R., Hermoso Carazo A., Linares-Pérez J, "Networked distributed fusion estimation under uncertain outputs with random transmission delays, packet losses and multi-packet processing,” Signal Processing, 2019, 156, 71-83.

[6] Lu F., Gao T.Y.Y., Huang J.Q, “ A novel distributed extended Kalman filter for aircraft engine gas-path health estimation with sensor fusion uncertainty,” Aerospace Science and Technology, 2019, 84, 90-106. 